\title{
Pós de rochas regionais como fonte de fósforo e potássio para plantas
}

\section{Regional rock dust as a source of phosphorus and potassium for plants}

Polvo de piedras regionales como fuentes de fosforo $y$ potasio para las plantas

Recebido: 06/05/2020 | Revisado: 10/05/2020 | Aceito: 14/05/2020 | Publicado: 23/05/2020

\section{Rafael Felippe Ratke}

ORCID: https://orcid.org/0000-0001-6930-3913

Universidade Federal de Mato Grosso do Sul, Brasil

E-mail: rafael.ratke@ufms.br

Thatiane Gomes Andrade

ORCID: https://orcid.org/0000-0003-0500-0862

Universidade Federal do Piauí, Brasil E-mail: thatiane.eng.agronoma@gmail.com

Sara Gomes da Rocha

ORCID: https://orcid.org/0000-0001-9499-2167

Universidade Federal do Piauí, Brasil

E-mail: saraagrogomes@ hotmail.com

Alan de Sousa

ORCID: https://orcid.org/0000-0002-5720-9521

Universidade Federal do Piauí, Brasil

E-mail: alandesousa161@gmail.com

Paulo Vinícius da Silva Dai

ORCID: https://orcid.org/0000-0002-0529-2566

Universidade Federal de Mato Grosso do Sul, Brasil

E-mail: pvsdai@hotmail.com

Edson Cavalcanti da Silva-Filho

ORCID: https://orcid.org/0000-0003-0988-2970

Universidade Federal do Piauí, Brasil

E-mail: edsonfilho@ufpi.edu.br 
Luiz Carlos Bertolino

ORCID: https://orcid.org/0000-0002-0908-180X Centro de Tecnologia Mineral, Brasil

E-mail: lcbertolino@cetem.gov.br

Alan Mario Zuffo

ORCID: https://orcid.org/0000-0001-9704-5325 Universidade Federal de Mato Grosso do Sul, Brasil

E-mail: alan_zuffo@hotmail.com

Augusto Matias de Oliveira

ORCID: https://orcid.org/0000-0003-3556-2030

Universidade Federal dos Vales do Jequitinhonha e Mucuri, Brasil

E-mail: augusto2013ufpi@gmail.com

Jorge González Aguilera

ORCID: https://orcid.org/0000-0002-7308-0967

Universidade Federal de Mato Grosso do Sul, Brasil

E-mail: j51173@yahoo.com

\section{Resumo}

Os pós de rochas contêm nutrientes que podem melhorar o desenvolvimento nutricional das plantas. Assim, objetivou-se avaliar a disponibilidade de fósforo $(\mathrm{P})$ e potássio $(\mathrm{K})$ através de pós de rochas coletados em diferentes locais e seus efeitos no desenvolvimento de plantas de milho. Utilizou-se três fontes de pós de rochas [coletados em Campo Alegre de Lourdes-BA (R1), Gilbués-PI (R2) e Cristalândia-PI (R3)], caracterizados por difratometria, granulometria, e pela dissolução de $\mathrm{P}$ e K em processo termodinâmico. Após a caracterização físico-químico dos pós, realizou-se a aplicação de diferentes doses (2,0; 6,0; 8,0 e 10,0 t ha-1) no solo para analisar seus efeitos em plantas de milho. Na rocha R1 observou-se muscovita (mica) e apatita (fosfatos), R2 apresenta muscovita (mica) e ortoclásio (feldspato) e R3 possui os minerais muscovita (mica) e microclínio. Os materiais rochosos apresentaram dissolução de $\mathrm{P}$ e $\mathrm{K}$ em meio ácido em extrato aquoso. As rochas coletadas em Gilbués-PI, melhorou a disponibilidade de $\mathrm{P}$ e $\mathrm{K}$, e favoreceu o desenvolvimento da parte aérea das plantas de milho.

Palavras-chaves: Rochagem; Difratometria; Granulometria; Zea mays. 
Abstract

Rock dust contains nutrients that can improve the nutritional development of plants. Thus, the objective was to evaluate the availability of phosphorus $(\mathrm{P})$ and potassium $(\mathrm{K})$ through rock powders collected in different locations and their effects on the development of corn plants. Three sources of rock powders were used [collected in Campo Alegre de Lourdes-BA (R1), Gilbués-PI (R2), and Cristalândia-PI (R3)], characterized by diffractometry, granulometry, and the dissolution of $\mathrm{P}$ and $\mathrm{K}$ in a thermodynamic process. After the physicochemical characterization of the powders, different doses $\left(2.0 ; 6.0 ; 8.0\right.$ and $\left.10.0 \mathrm{t} \mathrm{ha}^{-1}\right)$ were applied to the soil to analyze its effects on corn plants. In the rock R1, the presence of muscovite (mica) and apatite (phosphates) was observed, in R2 the presence of muscovite (mica) and orthoclase (feldspar) and in R3 the presence of muscovite (mica) and microcline. The rocky dust materials showed dissolution of $\mathrm{P}$ and $\mathrm{K}$ in an acid medium in the aqueous extract. The rocks dust collected in Gilbués-PI improved the availability of $\mathrm{P}$ and $\mathrm{K}$ and favored the development of the aerial part of the corn plants.

Keywords: Stonemeal; Diffractometry; Granulometry; Zea mays.

\section{Resumen}

Los polvos de roca contienen nutrientes que pueden mejorar el desarrollo nutricional de las plantas. Por lo tanto, el objetivo fue evaluar la disponibilidad de fósforo $(\mathrm{P})$ y potasio $(\mathrm{K})$ a través de los polvos de roca recolectados en diferentes lugares y sus efectos en el desarrollo de las plantas de maíz. Se utilizaron tres fuentes de polvo de roca [recolectadas en Campo Alegre de Lourdes-BA (R1), Gilbués-PI (R2) y Cristalândia-PI (R3)], caracterizadas por difractometría, granulometría y la disolución de $\mathrm{P}$ y $\mathrm{K}$ en proceso termodinámico. Después de la caracterización fisicoquímica de los polvos, se aplicaron diferentes dosis $(2.0 ; 6.0 ; 8.0 \mathrm{y}$ $10.0 \mathrm{t} \mathrm{ha}^{-1}$ ) al suelo para analizar sus efectos en las plantas de maíz. En la roca R1, se observó la presencia de moscovita (mica) y apatita (fosfatos), en R2 la presencia de moscovita (mica) y ortoclasa (feldespato) y en R3 la presencia de moscovita (mica) y microclina. Los materiales rocosos mostraron disolución de $\mathrm{P}$ y $\mathrm{K}$ en un medio ácido en extracto acuoso. Las rocas recolectadas en Gilbués-PI, mejoraron la disponibilidad de P y K, y favorecieron el desarrollo de la parte aérea de las plantas de maíz.

Palabras clave: Rocosidad; Difractometría; Granulometría; Zea mays. 


\section{Introdução}

O milho (Zea mays) é um cereal de importância socioeconômica mundial, cultivado em quase todos os países, utilizado na alimentação humana (in natura, indústrias de conservas alimentícias, processado) e animal (produção de silagem), destacando-se como uma importante fonte de alimento mundial, além de ser utilizado na produção de biogás (Daryanto et al., 2015; Ramirez-Cabral et al., 2017; Silva et al., 2017). O milho é uma importante fonte de renda para pequenos, médios e grandes produtores, que o cultiva em duas safras, normal e safrinha (Fuentes et al., 2018).

Perante sua importância, é comum estudos que visem melhorar sua produtividade, sendo o uso de fertilizantes minerais algo muito recorrido (Jing et al., 2019; Srivastava et al., 2019). No entanto, é uma tecnologia cara, não estando, portanto, acessível a todos os produtores, principalmente os mais pobres, sendo assim necessário a busca de fertilizantes alternativos como fonte de nutrientes para a cultura (Manning \& Theodoro, 2018). De acordo com Camargo et al. (2012), a aplicação de pó de rochas como fertilizantes químicos apresenta vantagem em relação ao custo reduzido e produtividade, diminuindo a demanda de fertilizantes convencionais, além de incrementar na velocidade de resposta do crescimento das plantas, melhorar a fertilidade do solo e mitiga impactos ambientais. Aguilera et al. (2020) ao avaliar cultivares de soja (Glycine max L. Merrill) nós quais foram aplicadas doses de pó de basalto observaram que estes contribuíram para obter um melhor tamanho das sementes e, assim, melhorar a qualidade das sementes obtidas.

Existe uma preocupação em relação ao uso ineficiente dos nutrientes minerais, que resulta em acúmulo, podendo gerar efeitos negativos ao meio ambiente, como aumento dos gases de efeito estufa e acúmulo em águas subterrâneas e costeiras (West et al., 2014). O fósforo, por exemplo, é um elemento pouco móvel no solo, assim, tem uma vida mais longa no ambiente de deposição, o que pode ocasionar degradação da qualidade da água, por exemplo (Swaney \& Howarth, 2019). Portanto, é necessário o uso eficiente da adubação mineral, pois os nutrientes também são essenciais para o bom desenvolvimento das culturas agrícolas.

O fósforo $(\mathrm{P})$ e o potássio $(\mathrm{K})$ são macronutrientes essenciais requeridos pelas plantas. O P integra importantes componentes das plantas, como açucares-fosfato, nucleotídeos fonte de energia (ATP), dentre outros compostos, auxiliando dessa maneira em processos 
fisiológicos e bioquímicos que auxiliam no crescimento e desenvolvimento vegetal (Li et al., 2017; Lu et al., 2016). O K está relacionado com a regulação osmótica das células, além de ser ativador de enzimas relacionadas a fotossíntese e respiração, desempenhando importantes funções em processos fisiológicos que também auxiliam no crescimento e desenvolvimento das plantas (Dreyer et al., 2017; Y. Wang \& Wu, 2017).

Assim, a rochagem (uso de pó de rochas moídas para a recuperação da fertilidade do solo) é uma técnica que além de gerar benefícios para as plantas e agricultores devido ser uma técnica mais barata, também reduz os danos ao meio ambiente, devido minimizar o acúmulo de nutrientes nos locais onde as rochas são extraídas (Manning \& Theodoro, 2018; Aguilera et al., 2020). Diante do exposto, objetivou-se com presente estudo avaliar a disponibilidade de $\mathrm{P}$ e $\mathrm{K}$ por meio de pós de rochas coletadas em diferentes locais e seus efeitos no desenvolvimento de plantas de milho.

\section{Material e Métodos}

O presente trabalho é uma pesquisa experimental quantitativa e qualitativa que segue os fundamentos desses tipos de pesquisa, conforme recomendado por Pereira et al., (2018). Parte dos dados quantitativos foi obtida em casa de vegetação e os qualitativos no laboratório.

A parte experimental foi desenvolvida em duas etapas: (1) caracterização do material rochoso por granulometria, fluorescência de raio X (FRX) e difratometria de raios X (DRX), e a dissolução de $\mathrm{P}$ e $\mathrm{K}$ do material rochoso na forma de pó em diferentes potenciais hidrogeniônicos $(\mathrm{pH})$ e temperaturas; (2) efeito das doses de diferentes pós de rochas na massa seca do milho e atributos químicos do solo. As amostras de materiais rochosos foram coletadas em três mineradoras localizadas em Campo Alegre de Lourdes-BA (Rocha 1 - R1), Gilbués-PI (Rocha 2 - R2) e Cristalândia-PI (Rocha 3 - R3).

\subsection{Caracterização dos materiais rochosos}

Antes da avaliação de solubilidade os materiais rochosos foram caracterizados fisicamente seguindo o método de peneiramento descrito no manual de métodos analíticos oficiais para fertilizantes e corretivos (Brasil, 2017), utilizando o delineamento inteiramente casualizado com três repetições. As peneiras foram empilhadas $(2 \mathrm{~mm} ; 0,85 \mathrm{~mm} ; 0,3 \mathrm{~mm}$; e 
prato retenção) com as aberturas das malhas crescendo de baixo para cima, e na parte superior da peneira de maior abertura foi colocada a tampa para evitar a perda de partículas no início do processo de vibração.

Utilizou-se $10 \mathrm{~g}$ dos materiais rochosos. Em seguida, as peneiras foram encaminhadas para o agitador de peneiras eletromagnético, onde permaneceram 10 minutos. Após a agitação, as frações de pó de rocha retida em cada peneira foram pesadas em balança analítica de precisão $(0,001 \mathrm{~g})$.

Parte das amostras em pó dos materiais rochosos foram homogeneizadas e encaminhadas para o Centro de Tecnologia Mineral (Ministério da Ciência, Tecnologia, Inovação e comunicações) no Rio de Janeiro, para a caracterização mineral do material rochoso em FRX e DRX. Foram pesados $5 \mathrm{~g}$ de cada amostra e posteriormente foi feita a perda por calcinação em duplicatas em mufla à uma temperatura de $1000^{\circ} \mathrm{C}$ para a análise em FRX, utilizando-se o espectrômetro de fluorescência de raios X por comprimento de onda, modelo Axios Max - Panalytical.

$\mathrm{O}$ difratograma de raios $\mathrm{X}$ das amostras foram obtidos pelo método do pó em um equipamento Bruker-D4 Endeavor, nas seguintes condições de operação: radiação CuKa (40 $\mathrm{kV} / 4040 \mathrm{~mA}$ ); velocidade do goniômetro de $0,02^{\circ} 2 \theta$ por passo com tempo de contagem de 0,5 segundos por passo e coletados de 4 a $80^{\circ} 2 \theta$, com detector sensível à posição LynxEye. A interpretação qualitativa do espectro foi efetuada por comparação com padrões contidos no banco de dados PDF02 (ICDD) em software Bruker DiffracPlus.

A avaliação da dissolução dos materiais rochosos em pó foi realizada no laboratório de solos do Campus Professora Cinobelina Elvas (CPCE), da Universidade Federal do Piauí

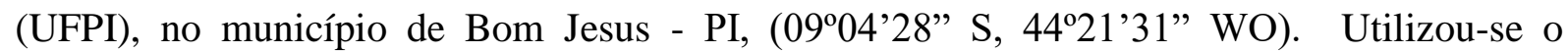
delineamento inteiramente casualizado com três repetições em esquema fatorial 3 x 5, sendo, três temperaturas $\left(25,45\right.$ e $\left.75^{\circ} \mathrm{C}\right)$ e cinco pHs $(3,0 ; 4,0 ; 5,0 ; 6,0$ e 7,0).

A alíquota de $1 \mathrm{mg}$ dos materiais rochosos foi colocada em Erlenmeyers de $250 \mathrm{ml}$, em soluções aquosas (água ultra-pura) com pH em água de 3,0; 4,0; 5,0; 6,0 e 7,0 (para realizar a acidificação das soluções utilizou-se $\mathrm{HCl}$ na concentração $1 \mathrm{~mol} \mathrm{~L}^{-1}$ e para alcalinizar $\mathrm{NaOH}$ na concentração $1 \mathrm{~mol} \mathrm{~L}^{-1}$ ) e temperaturas de 25,45 e $75^{\circ} \mathrm{C}$ em banho-maria por 30 minutos, posteriormente submetidos a decantação por 12 horas. Após esse período, foi coletado o sobrenadante para a análise dos teores de $\mathrm{P}$ e $\mathrm{K}$ disponíveis na solução. A análise de $\mathrm{P}$ foi realizada em espectrofotômetro de UV-visível e o de K em fotômetro de chama conforme o 
método proposto por Silva (2011), sendo as leituras nas soluções dos sobrenadantes, sem a utilização de extratos ácidos.

\subsection{Cultivo de milho com diferentes fontes e doses de pós de rochas}

O experimento foi realizado em casa de vegetação do CPCE-UFPI, em esquema fatorial $3 \times 4$ × $2+1$, sendo, três pós de rochas (R1, R2 e R3), em quatro doses (2,0; 6,0; 8,0 e 10,0 t

ha $^{-1}$, correspondente a $5 ; 12 ; 16$ e $20 \mathrm{~g} \mathrm{vaso}^{-1}$, respectivamente), com duas acidificações (com acidificação a pH 3,0 e sem acidificação da rocha antes da aplicação) mais uma testemunha adicional (sem adição do pós de rochas), conduzido em delineamento inteiramente casualizado com 3 repetições. Amostras dos pós de rochas foram colocadas em beckers de 1 litro e adicionado $500 \mathrm{~mL}$ de água deionizada, e em seguida homogeneizou-se a solução com bastão de vidro e aferiu-se o pH no potenciômetro. $\mathrm{O}$ pH da solução com pós de rocha foi ajustado para 3,0 através da adição de $\mathrm{HCl} \mathrm{a} 1 \mathrm{~mol} \mathrm{~L}^{-1}$. Posteriormente retirou os pós de rocha da solução ácida, deixando secar ao ar livre durante 48 horas e, posteriormente, aplicou-os em diferentes doses no solo dos vasos.

O solo utilizado no experimento foi coletado na profundidade de 0,20 m em área de mata nativa de cerrado situado na Serra das Laranjeiras, município de Currais, Piauí, classificado como Latossolo Amarelo distrófico (Pragana et al., 2012). O solo foi levado para laboratório de solos do CPCE-UFPI, sendo peneirado em malha de $2 \mathrm{~mm}$, seco ao ar e homogeneizado. O solo foi analisado físico-quimicamente seguindo a metodologia de Silva (2011).

A análise físico químico inicial apresentou os seguintes resultados $\mathrm{pH}(\mathrm{H} 2 \mathrm{O})$ : 3,6; P (Mehlich): $4 \mathrm{mg} \mathrm{dm}{ }^{-3} ; \mathrm{K}^{+}: 1,17 \mathrm{mg} \mathrm{dm}{ }^{-3} ; \mathrm{Ca}^{2+}: 0,3$ cmolc dm${ }^{-3} ; \mathrm{Mg}^{2+}: 0,1 \mathrm{cmolc} \mathrm{dm}^{-3} ; \mathrm{Al}: 0,5$ cmolc dm ${ }^{-3}$; (H+Al): 5,8 cmolc dm ${ }^{-3}$; SB: 0,43 $\mathrm{cmolc} \mathrm{dm}^{-3}$; CTC potencial: $6,24 \mathrm{cmolc} \mathrm{dm}^{-3}$; V: 6,89\%; m: 54,00\%; Matéria orgânica: $2,5 \mathrm{~g} \mathrm{~kg}^{-1}$; argila: $167 \mathrm{~g} \mathrm{~kg}^{-1}$, silte: $17 \mathrm{~g} \mathrm{~kg}^{-1} \mathrm{e}$ areia: $816 \mathrm{~g} \mathrm{~kg}^{-1}$ ). De acordo com Ratke et al. (2018), verificou-se a necessidade de calagem decorrente a alta acidez conforme os resultados da análise de solo. Porém, os pós de rochas utilizados podem apresentar elementos básicos como $\mathrm{SiO}, \mathrm{CaO}$ e $\mathrm{MgO}$ (Ramos et al. 2014). Dessa forma, avaliou-se a eficiência na correção da acidez do solo em função da aplicação dos pós de rocha ao final do experimento. 
Foram semeadas quatro sementes de milho (Cv. 2B810) por vaso e 10 dias após a germinação (DAG) foi feito o raleio deixando somente duas plantas por vaso. Realizou-se uma adubação com $\mathrm{N}$ nessa fase (267 mg por vaso de ureia, o que corresponde a $120 \mathrm{~kg} \mathrm{ha}^{-1}$ ), dividida em duas etapas, aos 15 e aos 30 dias. Utilizou-se uma solução nutritiva de Hoagland \& Arnon (1950) de macro e micronutrientes para a adubação do milho, suprimindo P e K, aplicando-se $4 \mathrm{~mL}$ desta solução aos 10 e 20 DAG. A irrigação consistiu em manter o solo em $60 \%$ da capacidade de campo, repondo-se a perda de água quando necessário, verificado através por meio de pesagem dos vasos com balança de precisão $(0,001 \mathrm{~g})$.

O solo dos vasos e as plantas de milho foram coletados aos 60 DAG. Antes de retirar as plantas, coletou-se $200 \mathrm{~g}$ de solo de cada vaso com uma pequena pá em quatro pontos aleatórios do vaso, e colocados em sacos plásticos. Posteriormente, as amostras solos foram homogeneizadas, peneirado em peneira de $2 \mathrm{~mm}$ e seco ao ar. Analisou-se quimicamente nessas amostras: $\mathrm{pH}\left(\mathrm{H}_{2} \mathrm{O}\right), \mathrm{P}$ e $\mathrm{K}$ (Melhich1) seguindo a metodologia de Teixeira et al., (2017).

As plantas de milho foram cortadas rente ao solo para mensurar a massa seca de raiz e parte aérea. As raízes foram lavadas em água corrente para a retirar o solo aderido. Em seguida, a parte área e as raízes foram lavadas em água destilada para retirar a poeira e detritos, parcialmente secas em papel toalha e colocadas em sacos de papel. Depois foram levadas para secagem em estufa por 72 horas a $65{ }^{\circ} \mathrm{C}$, até atingir peso constante. A massa seca de raiz (MSR) e da parte aérea (MSPA) foram aferidas em balança analítica de precisão $(0,0001 \mathrm{~g})$.

\subsection{Análise estatística}

Os resultados da solubilidade, de produção de massa seca do milho e da análise de solo foram submetidos à análise de variância (teste $\mathrm{F}, P<0,05$ ). Quando significativo, as médias foram comparadas pelo teste de tukey $(P<0,05)$, confeccionado gráfico de Box-Plot e realizada análise canônica para identificação da relação massa seca de plantas de milho com a química do solo e o uso de pó de rocha, utilizando o software Rbio (Bhering, 2017). Os resultados quantitativos em função da interação, doses, acidez e rocha foram submetidos análise por regressão linear e polinomial, e comparados pelo teste de tukey $(P<0,05)$. 


\section{Resultados e Discussão}

Nesta seção se apresenta os resultados obtidos nos experimentos realizados ao caracterizar a parte física e química de três pós de rochas provenientes de locais diferentes e avaliadas a sua aplicação no desenvolvimento de plantas de milho.

\subsection{Caracterização físico química das rochas}

Os materiais rochosos apresentaram mais de $50 \%$ das partículas inferiores a $0,3 \mathrm{~mm}$, sendo, portanto, classificados como pó (Brasil, 2017) (Tabela 1).

Tabela 1. Retenção de partículas dos materiais rochosos em diferentes peneiras.

\begin{tabular}{|c|c|c|c|c|c|c|}
\hline \multirow{2}{*}{ Material* } & \multirow{2}{*}{ Unidade } & \multicolumn{3}{|c|}{ Abertura da peneira $(\mathrm{mm})$} & \multirow{2}{*}{ Prato } & \multirow{2}{*}{$\mathrm{CV}(\%)$} \\
\hline & & 2,00 & 0,85 & 0,30 & & \\
\hline \multirow{2}{*}{ R1 } & $\mathrm{g}$ & 0,01 & 0,83 & 3,34 & 5,82 & \multirow{2}{*}{2,63} \\
\hline & $\%$ & 0,10 & 8,30 & 33,40 & 58,20 & \\
\hline \multirow{2}{*}{$\mathrm{R} 2$} & $\mathrm{~g}$ & 0,03 & 0,82 & 3,98 & 5,17 & \multirow{2}{*}{2,47} \\
\hline & $\%$ & 0,30 & 8,20 & 39,80 & 51,70 & \\
\hline \multirow{2}{*}{ R3 } & $\mathrm{g}$ & 0,00 & 0,00 & 0,56 & 9,44 & \multirow{2}{*}{4,63} \\
\hline & $\%$ & 0,00 & 0,00 & 5,60 & 94,40 & \\
\hline
\end{tabular}

*Material: R1- Rocha 1 (Campos Alegre de Lourdes - BA); R2 - Rocha 2 (Gilbués - PI); R3 - Rocha 3 (Cristalândia - PI); CV - Coeficiente de variação. Fonte: Própria dos autores.

Apesar de todos os materiais serem classificados como pó, $94 \%$ das partículas de R3 não foram retidas na peneira de $0,3 \mathrm{~mm}$, enquanto somente 58 e $52 \%$ das partículas do R1 e R2, respectivamente, passaram nessa mesma peneira. Segundo Aguilera et al. (2020), a produção de soja foi maior com o uso de pós de rocha com partículas de 6,5 $\mathrm{mm}$ de diâmetro. Os materiais provenientes de rochas utilizados na agricultura como fonte de nutrientes apresentam tamanho de partículas diferentes, como os materiais calcários (Quaggio, 2000; Viadé et al., 2011). Nesse sentido, os pós de rochas apresentaram tamanhos de partículas diferentes, e fator que influência em sua solubilidade.

Assim, R3 pode apresentar maior solubilidade que R1 e R2, pois quanto menor a granulometria mais solúvel tende a ser o material. A dissociação de elementos químicos de menor granulometria é mais rápida, enquanto os de maior granulometria tem uma reação mais lenta, indicando assim que R3 tem alta solubilidade e baixo poder residual no solo quando comparado ao R1 e R2. Segundo Ratke et al. (2018), o uso de pó de rocha calcária com partículas menor $0,3 \mathrm{~mm}$ tem maior solubilidade e menor poder residual no solo. 
A presença de elementos químicos em pós de rochas depende de sua formação geológica e está relacionado com sua composição mineralogia (Ramos et al., 2014). Nesse sentido, os pós de rochas apresentaram diferentes níveis de elementos químicos: R1 possui maior concentração de $\mathrm{MgO}, \mathrm{P}_{2} \mathrm{O}_{5}, \mathrm{CaO}, \mathrm{MnO}, \mathrm{SrO}, \mathrm{BaO}, \mathrm{CeO}_{2}, \mathrm{~F}, \mathrm{La}_{2} \mathrm{O}_{3}$ e $\mathrm{Nd}_{2} \mathrm{O}_{3}$, e menor teor de silício; $\mathrm{R} 2$ possui maiores teores de $\mathrm{Al}_{2} \mathrm{O}_{3}, \mathrm{SiO}_{2}, \mathrm{~K}_{2} \mathrm{O}$, além de uma maior perda por calcinação, enquanto que $\mathrm{R} 3$ deteve maiores valores de $\mathrm{Na}_{2} \mathrm{O}, \mathrm{TiO}_{2}$ e $\mathrm{Fe}_{2} \mathrm{O}_{3}$ (Tabela 2). Dessa forma, os pós de rochas são classificados como fertilizantes multinutrientes, por fornecer mais de um nutriente para a planta, como descrito por Straaten (2006). Os pós de rocha R1e R2 são fertilizantes potenciais para fornecer $\mathrm{P}$ e $\mathrm{K}$ para plantas.

Tabela 2. Características químicas dos diferentes materiais rochosos analisados em FRX.

\begin{tabular}{|c|c|c|c|}
\hline \multirow{2}{*}{ Elemento/Mineral } & \multicolumn{3}{|c|}{ Material* } \\
\hline & $\mathrm{R} 1 *$ & R2 & R3 \\
\hline & & $---\%-$ & \\
\hline $\mathrm{Na}_{2} \mathrm{O}$ & 0,30 & 0,18 & 4,60 \\
\hline $\mathrm{MgO}$ & 4,70 & 3,80 & 2,40 \\
\hline $\mathrm{Al}_{2} \mathrm{O}_{3}$ & 4,20 & 16,40 & 15,90 \\
\hline $\mathrm{SiO}_{2}$ & 11,00 & 61,10 & 61,10 \\
\hline $\mathrm{P}_{2} \mathrm{O}_{5}$ & 32,00 & 0,23 & 0,50 \\
\hline $\mathrm{SO}_{3}$ & $<0,1$ & $<0,1$ & $<0,1$ \\
\hline $\mathrm{K}_{2} \mathrm{O}$ & 0,70 & 2,80 & 2,00 \\
\hline $\mathrm{CaO}$ & 39,10 & 3,80 & 2,70 \\
\hline $\mathrm{TiO}_{2}$ & 0,24 & 0,58 & 1,10 \\
\hline $\mathrm{MnO}$ & 0,10 & $<0,1$ & $<0,1$ \\
\hline $\mathrm{Fe}_{2} \mathrm{O}_{3}$ & 2,80 & 4,30 & 7,60 \\
\hline $\mathrm{SrO}$ & 0,91 & $<0,1$ & $<0,1$ \\
\hline $\mathrm{BaO}$ & 0,19 & $<0,1$ & $<0,1$ \\
\hline $\mathrm{CeO}_{2}$ & 0,16 & $\mathrm{ND}^{* * *}$ & ND \\
\hline $\mathrm{F}$ & 1,40 & ND & ND \\
\hline $\mathrm{La}_{2} \mathrm{O}_{3}$ & 0,15 & ND & ND \\
\hline $\mathrm{Nd}_{2} \mathrm{O}_{3}$ & 0,20 & ND & ND \\
\hline $\mathrm{PPC}^{* * *}$ & 1,60 & 6,60 & 1,90 \\
\hline
\end{tabular}

*Material: R1- Rocha 1 (Campos Alegre de Lourdes - BA); R2 - Rocha 2 (Gilbués - PI); R3 - Rocha 3 (Cristalândia - PI); **PPC - Perdas por Calcinação; ***ND - Não determinado. Fonte: Própria dos autores.

A análise de DRX revelaram a presença de vários minerais nos pós de rochas, estando os minerais silicatados em abundância (Figura 1). A classe dos minerais silicatados constituem $40 \%$ dos minerais comuns, constituindo mais de $90 \%$ da crosta terrestre (Klein \& Dutrow, 2012). As predominâncias dos minerais silicatados nos pós de rochas foram de mica, esmectita, vermiculita e principalmente feldspato, as análises apresentaram mineral não silicatado, como a albita. Em R1 foram identificados os seguintes minerais: muscovita, quartzo, barita, apatita, montmorillonita, vermiculita, o que indica a presença dos elementos $\mathrm{K}$ (muscovita - mica) e P (apatita - fosfatos) (Figura 1A). No R2 foi verificada os minerais muscovita, quartzo, albita e ortoclásio, demonstrando dessa maneira a presença de $\mathrm{K}$ 
(CC BY 4.0) | ISSN 2525-3409 | DOI: http://dx.doi.org/10.33448/rsd-v9i7.4257

(Muscovita - mica e ortoclásio - feldspato) (Figura 1B). Os minerais montmorilonita, quartzo, muscovita e microclínio foram identificados em $\mathrm{R} 3$, constituído por $\mathrm{K}$ e $\mathrm{SiO}_{2}$ (muscovita - mica e microclínio) (Figura 1C). Os minerais muscovita, mica, apatita, montmorilonita e vermiculita apresenta-se muitos reativos, e com isso um potencial de uso agrícola como fonte de nutrientes (Ramos et al., 2014).

Figura 1. Resultados de difratogrametria de raios $X$ dos materiais rochosos R1 (A), R2 (B) e R3 (C). Radiação Cu Ka (40 kV/40 mA).

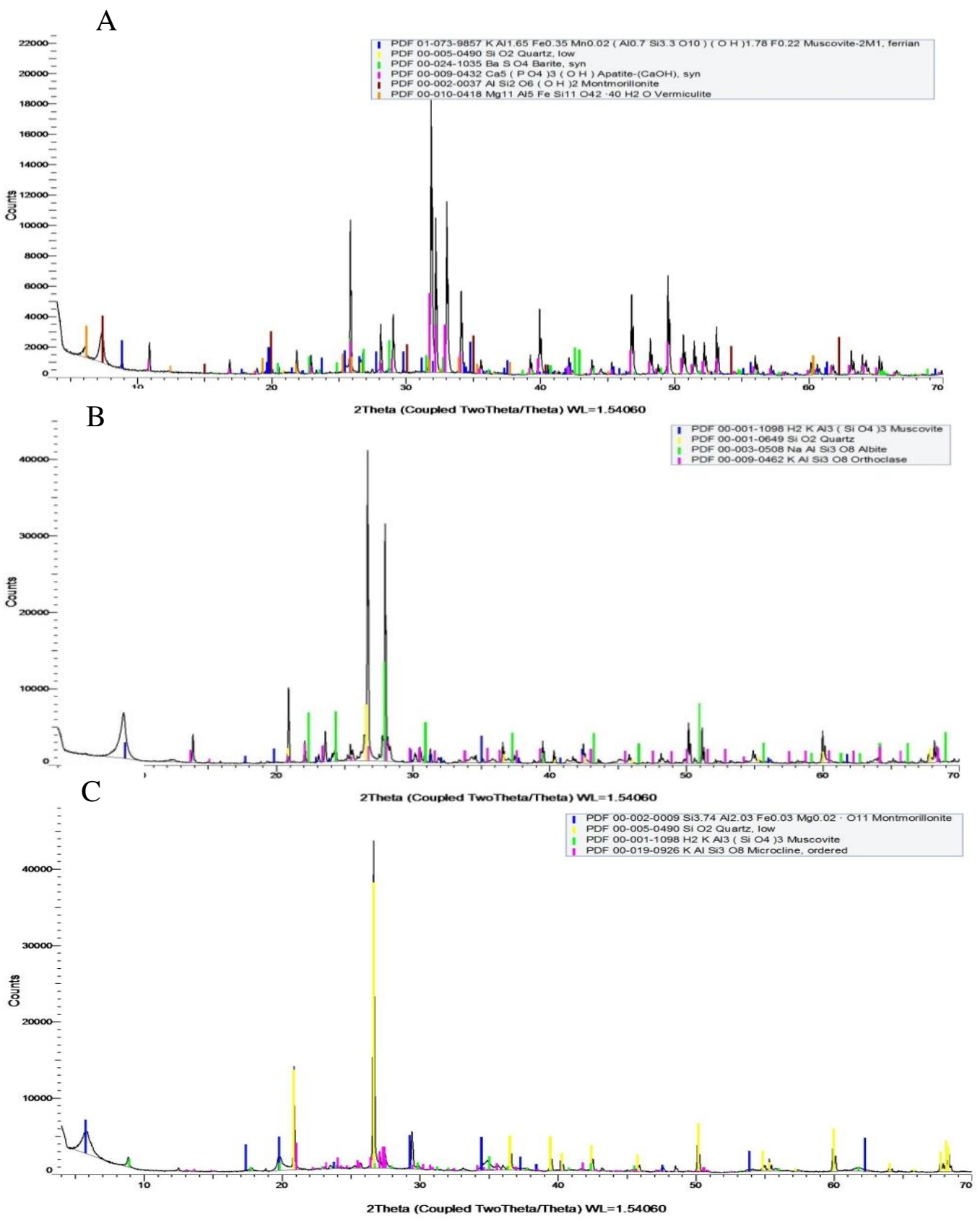

Fonte: Própria dos autores. 
(CC BY 4.0) | ISSN 2525-3409 | DOI: http://dx.doi.org/10.33448/rsd-v9i7.4257

Figura 2. Teores de $\mathrm{K}$ e $\mathrm{P}$ em água em diferentes temperaturas e $\mathrm{pH}$ aplicados nos pós de rochas R1 (A e B), R2 (C e D) e R3 (E e F). Equação significativa pelo teste $\mathrm{F}$ ( ${ }^{*} \mathrm{P}$ < $0,01 ; * * P<0,05)$; ns: não significativa.

$\mathrm{K}\left(\mathrm{mg} \mathrm{L}^{-1}\right)$

A) $\mathrm{R} 1-\mathrm{K}=36,88-0,07 \mathrm{x}-2,66 \mathrm{y} \mathrm{R}^{2}=0,56 *$

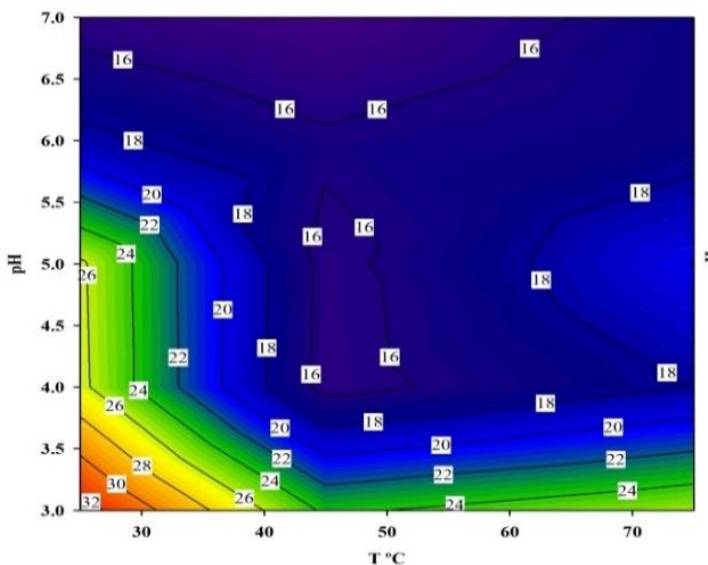

C) $\mathrm{R} 2-\mathrm{K}=62,64+0,06 \mathrm{x}-3,16 \mathrm{y} \mathrm{R}^{2}=0,28^{\mathrm{ns}}$



E) $R 3-K=4,65-0,06 x-5,53 y R^{2}=0,74 *$

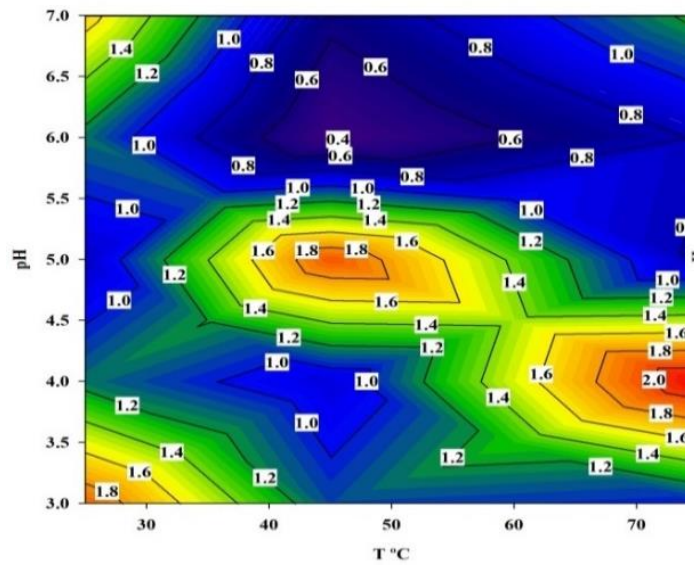

$\mathrm{P}\left(\mathrm{mg} \mathrm{L}^{-1}\right)$

B) $\mathrm{R} 1-\mathrm{P}=78,09+0,06 \mathrm{x}-12,42 \mathrm{y} \mathrm{R} \mathrm{R}^{2}=0,48 * *$

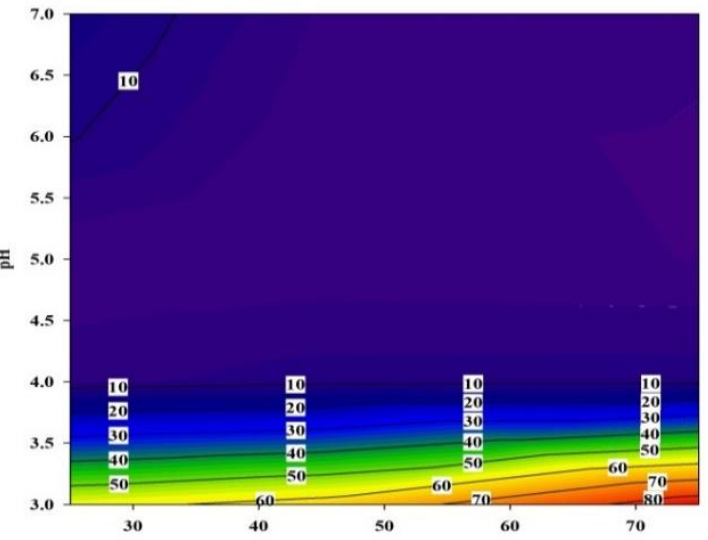

D) $\mathrm{R} 2-\mathrm{P}=2,64-0,001 \mathrm{x}-0,21 \mathrm{y} \mathrm{R}^{2}=0,21^{\mathrm{ns}}$

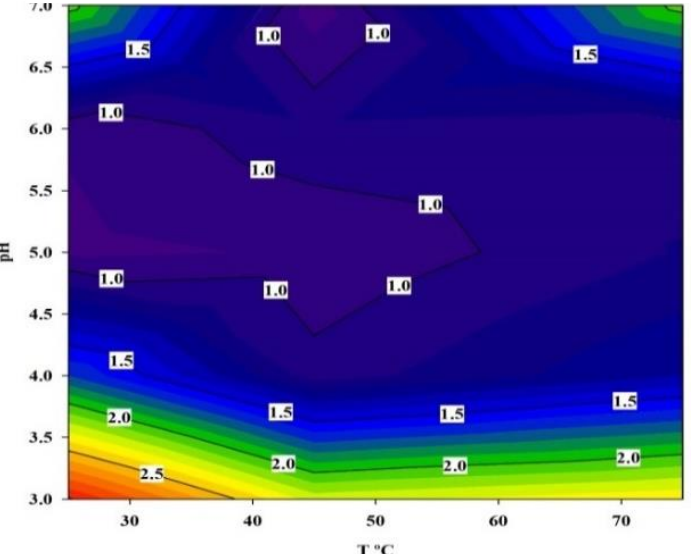

F) R3 - P $=1,74-0,002 x-0,09 y R^{2}=0,07^{\text {ns }}$

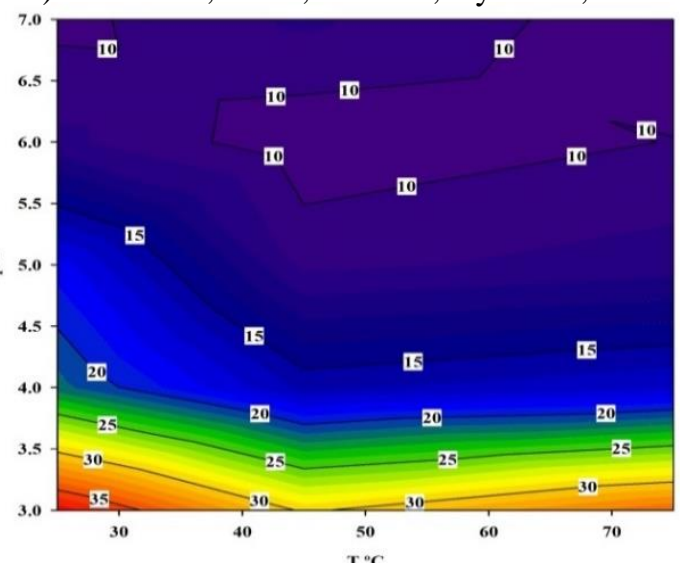

Fonte: Própria dos autores.

A dissociação de $\mathrm{P}$ e $\mathrm{K}$ dos pós de rocha foram influenciados pelo uso de diferentes temperaturas e pHs (Figura 2). $\mathrm{O}$ pH mais ácido promove maior disponibilidade de $\mathrm{K}$ em 
água nos pós de rochas extraídos de R1 e R3, sendo pouco independente da temperatura testada (Figura 2A e 2E). O R2 não apresentou efeito significativo para a dissociação de K em água em função de temperatura e pH (Figura 2C). Entretanto, o maior valor de dissolução K, $70,3 \mathrm{mg} \mathrm{L}^{-1}$, em água, foi observado no pó de rocha $\mathrm{R} 2$, no tratamento de $\mathrm{pH} 3,0$ a $25^{\circ} \mathrm{C}$. $\mathrm{O}$ $\mathrm{R} 2$ torna-se promissora fonte de $\mathrm{K}$ sem tratamentos prévios para a agricultura. Entretanto, Ribeiro et al. (2010), relatam que a dissolução de K de rochas silicáticas não apresentou influência do pH. A elevação da temperatura promoveu a dissolução de P no R1 (Figura 2B). Contudo, o R2 e o R3 não apresentaram respostas significativas para dissociação de P em água elevando-se a temperatura e pH (Figura 2D e 2F). O maior teor de $\mathrm{P}$ dissociado, 85,37 $\mathrm{mg} \mathrm{L} \mathrm{L}^{-1}$, foi obtido no $\mathrm{R} 1$ na temperatura $75^{\circ} \mathrm{C}$ em $\mathrm{pH} 3,0$. Assim, o R1 torna-se uma fonte $\mathrm{P}$, sendo que o tratamento térmico favorece sua solubilidade e pode disponibilizar mais $\mathrm{P}$ ao solo.

\subsection{Influência nos atributos químicos do solo e variáveis agronômicas de milho}

Os pós de rochas influenciaram significativamente o $\mathrm{pH}$ do solo, o $\mathrm{P}$ do solo, e a MSR e a MSPA do milho (Tabela 3). Os teores de pH do solo apresentaram efeito significativo para interação Rocha (R), Acidulação (A) e doses (D). Os fatores R, A e a interação A x D promoveram efeito significativo para os teores de $\mathrm{P}$ no solo. A interação dos fatores $\mathrm{R} \times \mathrm{A}$ influenciaram os valores de MSR de milho. Estes resultados corroboram com os que descreveram Ribeiro et al., (2010), que observaram aumento nos teores de P e K do solo utilizar pós de rochas ultramáfica alcalina e brecha piroclástica, e sendo que a rocha ultramáfica promoveu a elevação do $\mathrm{pH}$ do solo. Nesse sentido, observou-se que a adubação de milho com pós de rochas (Fatorial) aumentaram os teores de pH e P no solo ao comparálos com a testemunha (Adicional) sem aplicação de fertilizantes.

As diferentes doses de pós de rochas, acidulado e não acidulados aumentaram os teores de $\mathrm{pH}$ do solo quando comparado com o tratamento sem adubação (testemunha adicional) (Figura 3). Os pós de rochas R1, quando acidificado, e os pós de rocha R2, R3 não acidificado, promoveram aumento do $\mathrm{pH}$ do solo, apresentando regressão linear significativa para as suas respectivas doses (Figura 3A e Figura 3B). Os teores de $\mathrm{pH}$ do solo promoveram regressão polinomial significativa para doses do pó de rocha $\mathrm{R} 2$, e não significativa para doses do pó de rocha R3 (Figura 3A). Nesse sentido, observa-se que o aumento do pH no solo 
Research, Society and Development, v. 9, n. 7, e497974257, 2020

(CC BY 4.0) | ISSN 2525-3409 | DOI: http://dx.doi.org/10.33448/rsd-v9i7.4257

com o uso de pós de rochas $\mathrm{R} 2$ e R3, é justificado pela presença de $\mathrm{SiO}_{2}$ na sua composição mineralógica em comparação a R1 (Tabela 2), e a acidificação não promove a sua liberação, o que, ao contrário ocorreu na R1. Segundo Menegale et al. (2015), os materiais contendo o íon silicato $\left(\mathrm{SiO}_{2}\right)$ em sua composição, promovem a elevação do $\mathrm{pH}$ do solo, portanto, podem ser utilizados como corretivos de acidez do solo.

Tabela 3. Análise de variância dos teores de pH, P e K do solo e MSR e MSPA de milho.

\begin{tabular}{|c|c|c|c|c|c|}
\hline Fontes de variação & $\mathrm{pH}$ & $\mathrm{P}$ & $\mathrm{K}$ & MSR & MSPA \\
\hline & \multicolumn{5}{|c|}{ 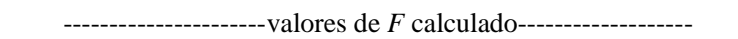 } \\
\hline Rocha (R) & $9,45^{*}$ & $27,72 *$ & $0,29^{\text {ns }}$ & $2,87^{\text {ns }}$ & $21,67 *$ \\
\hline Acidificação (A) & $0,05^{\mathrm{ns}}$ & $0,53^{\mathrm{ns}}$ & $0,18^{\mathrm{ns}}$ & $3,56^{\mathrm{ns}}$ & $0,36^{\mathrm{ns}}$ \\
\hline Dose (D) & $3,24^{\mathrm{ns}}$ & $3,62^{\text {ns }}$ & $1,45^{\mathrm{ns}}$ & $0,83^{\text {ns }}$ & $1,87^{\mathrm{ns}}$ \\
\hline $\mathrm{R} \times \mathrm{A}$ & $7,81 *$ & $18,38^{*}$ & $0,87^{\mathrm{ns}}$ & $3,39 *$ & $1,03^{\mathrm{ns}}$ \\
\hline $\mathrm{R} \times \mathrm{D}$ & $2,33 * *$ & $1,48^{\mathrm{ns}}$ & $1,35^{\mathrm{ns}}$ & $1,23^{\mathrm{ns}}$ & $0,41^{\mathrm{ns}}$ \\
\hline$A \times D$ & $1,12^{\mathrm{ns}}$ & $4,20 *$ & $1,54^{\mathrm{ns}}$ & $0,65^{\mathrm{ns}}$ & $0,61^{\mathrm{ns}}$ \\
\hline$R \times A \times D$ & $6,05^{*}$ & $1,68^{\text {ns }}$ & $1,17^{\mathrm{ns}}$ & $1,85^{\mathrm{ns}}$ & $0,49^{\text {ns }}$ \\
\hline Adicional versus fatorial & $6,38^{*}$ & $4,48^{* *}$ & $0,21^{\mathrm{ns}}$ & $1,24^{\mathrm{ns}}$ & $2,52^{\mathrm{ns}}$ \\
\hline \multirow[t]{2}{*}{ Tratamentos } & $\mathrm{pH}$ & $\mathrm{P}$ & $\mathrm{K}$ & & \\
\hline & $\mathrm{H}_{2} \mathrm{O}$ & $\mathrm{mg} \mathrm{dm}^{-3}$ & $\mathrm{mg} \mathrm{dm}^{-3}$ & g planta $^{-1}$ & g planta $^{-1}$ \\
\hline adicional & $4,16 b$ & $4,91 \mathrm{~b}$ & 24,00 & 0,12 & 1,51 \\
\hline fatorial & $4,54 \mathrm{a}$ & $16,83 \mathrm{a}$ & 25,91 & 0,21 & 1,84 \\
\hline Coeficiente de variação (\%) & 8,65 & 38,67 & 6,60 & 25,88 & 17,18 \\
\hline
\end{tabular}

* e ** Significativo a $1 \%$ e $5 \%$, respectivamente; ns: não significativo; Letras minúsculas nas colunas não diferem entre si pelo teste de Tukey $(P<0.05)$. pH: potencial hidrogeniônico; P: fósforo; K: potássio; MSR: massa seca de raiz; MSPA: massa seca da parte aérea. Fonte: Própria dos Autores.

Figura 3. Teores de pH do solo em função de doses de Pós de Rochas Acidificadas (A) e Não Acidificadas (NA). R1: Campo Alegre de Lourdes-BA; R2: Gilbués-PI e R3: Cristalândia-PI. Símbolos seguidas de mesma letra minúscula não diferem entre si pelo teste de Tukey $(P<0,05)$. Equação significativa pelo teste $\mathrm{F}\left({ }^{*} P<0,01 ; * * P<0,05\right)$; ns: não significativa.

A

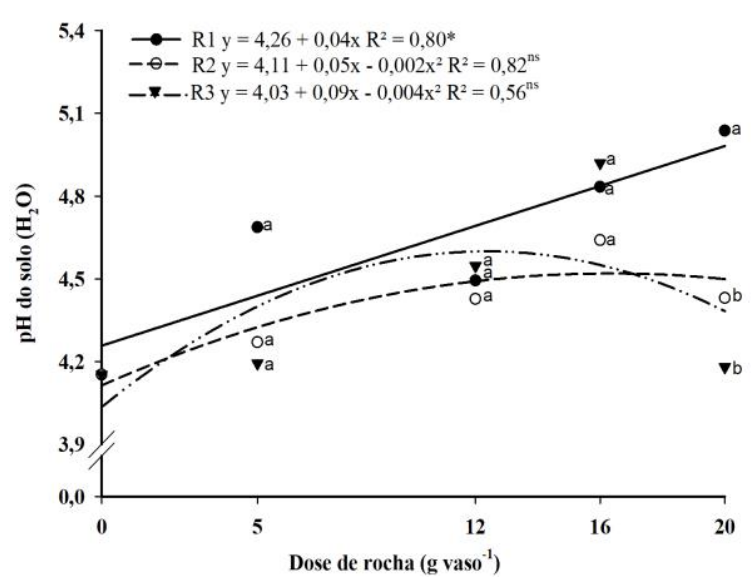

B

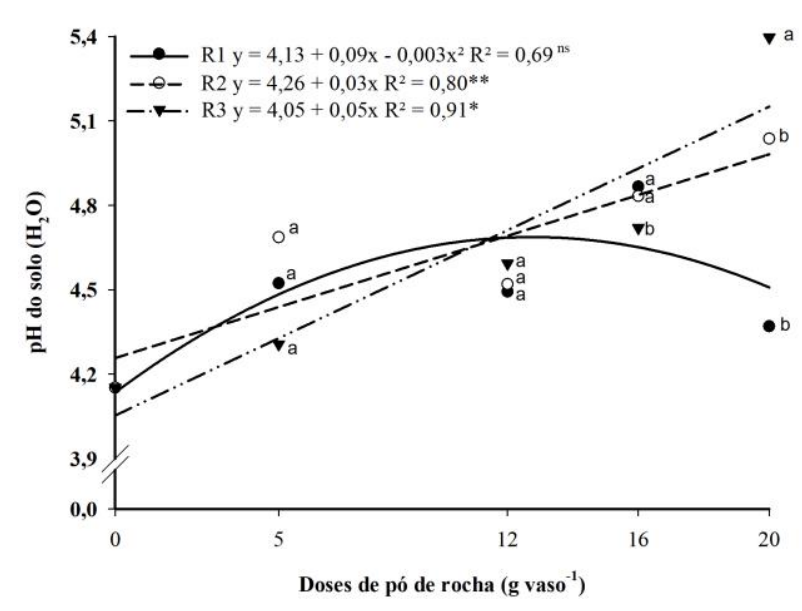

Fonte: Própria dos autores 
As doses de pós de rochas acidificados (PRA) promoveram aumentos nos teores de $\mathrm{P}$ do solo apresentando equação linear significativa (Figura 4). A presença de $\mathrm{CaO}$ de $\mathrm{MgO}$ nos pós de rocha indicam que o $\mathrm{P}$ estava complexado com $\mathrm{CaO}$, e que ao acidificar o meio promoveu a liberação do fosfato (Ramos et al., 2014). Porém, isso ocorreu no pó de rocha R1, devido a presença de Apatita neste material. Entretanto, as R2 e R3, aumentou a disponibilidade de fósforo no solo, devido ao alto teor de $\mathrm{SiO}_{2}$. O fosfato e o silicato são adsorvidos no mesmo sítio coloidal do solo, havendo, portanto, o deslocamento do fosfato da fase sólida para líquida, quando aumenta-se o teor de silicato no solo (Santos et al., 2008; Schaller et al., 2019). Nesse sentido, materiais que possuem silicato promovem o aumento da disponibilidade de P no solo (Menegale et al. 2015).

Figura 4. Teores de P do solo em função de doses de Pós de Rochas Acidificadas (PRA) e Não Acidificadas (PRNA). Símbolos seguidas de mesma letra minúscula não diferem entre si pelo teste de Tukey $(P<0,05)$. Equação significativa pelo teste $\mathrm{F}(* P<0,01$; $* * P<0,05)$; ns: não significativa.

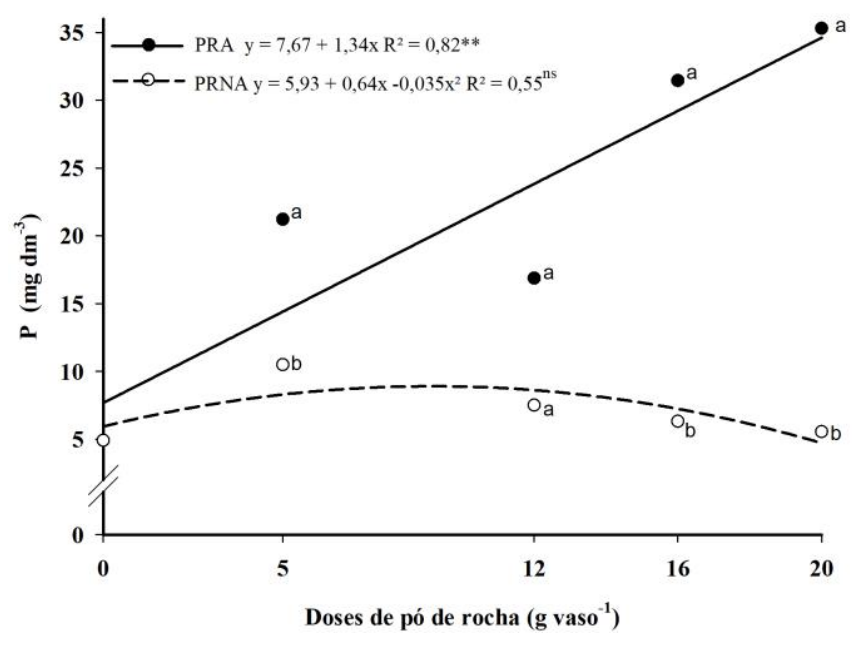

Fonte: Própria dos autores

A aplicação de pós de rochas R1 e R2 promoveram maior MSPA do milho, não se diferindo significativamente entre si, entretanto, foram superiores aos tratamentos R3 e o tratamento sem adubação (Figura 5A). A diminuição da MSR de milho foi proporcionada pela aplicação de pó de rocha R2 acidificados, igualando-se ao tratamento sem adubação (Figura 5B). Os pós de rochas R1 e R3 proporcionou maiores valores de MSR, independente da acidificação prévia, em comparação a R2 e ao tratamento sem adubação. Segundo Melo et al. 
(2018), a produção de capim Massai apresentou efeitos positivos do uso de pós de rocha como fonte de fósforo. Os materiais que contém silicatos aumentam a produção de massa seca das culturas, principalmente gramíneas forrageiras (Menegale et al., 2015). Assim, os pós de rochas são uma alternativa nutrientes e, principalmente, para gramíneas.

Figura 5. Box-plot MSPA - massa seca da parte aérea (A) e MSR - massa seca de raiz (B) em função da aplicação de pós de rochas. R1: Campo Alegre de Lourdes-BA; R2: Gilbués-PI e R3: Cristalândia-PI. TEST - tratamento controle. AC: Acidificadas; NA: Não Acidificados. Médias seguidas de mesma letra minúscula acima das barras não diferem entre si pelo teste de Tukey $(P<0,05)$.
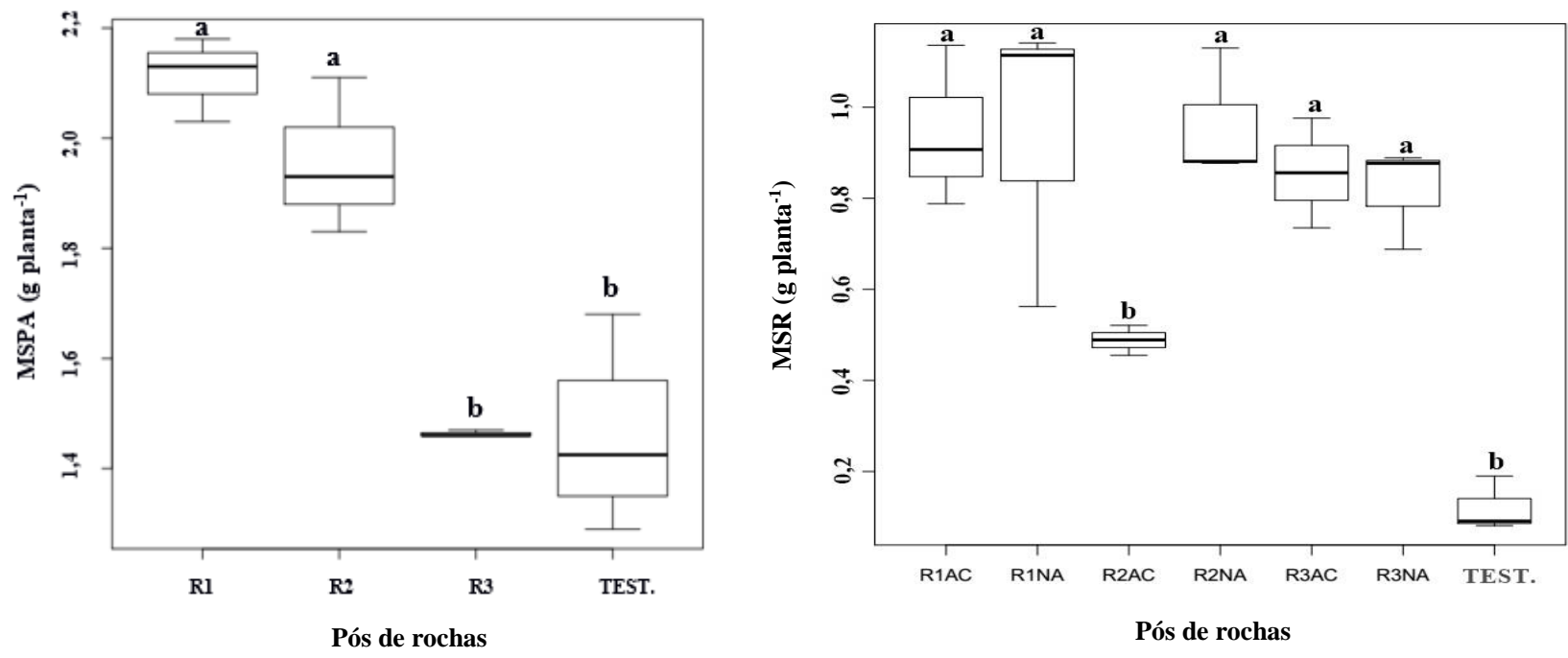

Fonte: Própria dos autores

O pó de rocha R1, apresentou forte correlação para a MSR de milho e o $\mathrm{pH}$ do solo, enquanto R2 correlações com o teor de $\mathrm{P}$ e K no solo e MSPA, porém o pó de rocha R3 ficou num quadrante isolado, mostrando pouco efeito sobre as variáveis analisadas (Figura 6). A disponibilidade de $\mathrm{P}$ promovida pela adição dos pós de rochas influenciam o desenvolvimento e produção das plantas. De acordo com Aguilera et al. (2020), o uso de pós de rochas influenciou os parâmetros produtivos de soja. Dessa forma, fontes alternativas de $\mathrm{P}$ podem ser empregados para a produção de culturas como é o caso de milho e soja.

O P e o K são elementos essenciais para o crescimento e desenvolvimento das plantas. Portanto, são macronutrientes que estão diretamente ligados aos processos físiológicos e bioquímicos das plantas. Assim, quanto melhor a disponibilidade desses, melhor será o crescimento e desenvolvimento das plantas, acarretando, consequentemente, em melhor desenvolvimento da parte área das culturas e em sua massa seca. O uso de pós de rochas são 
uma alternativa para o fornecimento de $\mathrm{P}$ e $\mathrm{K}$ para cultura do milho, e esta apresenta-se beneficiada quanto utilizados pós de rochas silicatados, pois o silício é um elemento benéfico, principalmente para gramíneas forrageiras (Menegale et al., 2015).

Figura 6. Gráfico de análise canônica de MSPA (massa seca parte aérea), MSR (massa seca de raiz), $\mathrm{pH}$ do solo, e $\mathrm{P}$ e $\mathrm{K}$ disponível no solo em função do uso de pó de rochas R1: Campo Alegre de Lourdes-BA; R2: Gilbués-PI e R3: Cristalândia-PI.

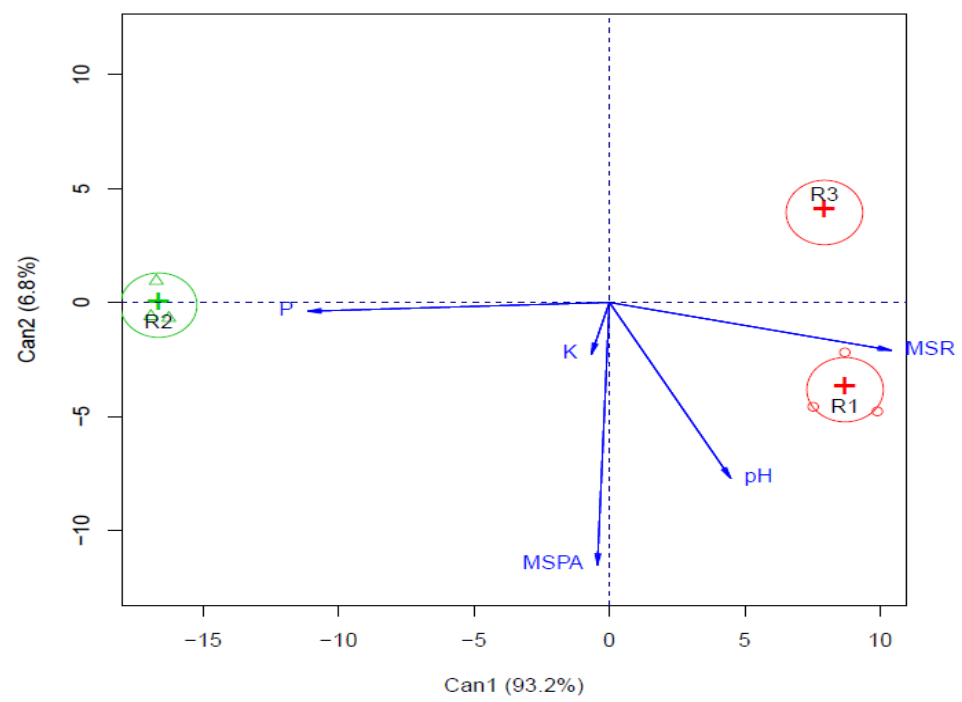

Fonte: Própria dos autores

\section{Conclusão}

Os tratamentos termodinâmicos de temperatura e $\mathrm{pH}$ promove a dissolução de $\mathrm{K}$ nos pós de rochas.

$\mathrm{O}$ uso de pós de rocha regionais como fonte $\mathrm{K}, \mathrm{P}$ e corretivo da acidez do solo, sendo uma alternativa eficiente para melhorar a fertilidade do solo.

A aplicação de pós de rochas R1 (Lourdes) previamente acidificado, R2 (Cristalândia) e R3 (Gilbués) não acidificado promovem o aumento do pH do solo

A aplicação de pós de rochas previamente acidificados promove o aumento do teor de $\mathrm{P}$ no solo.

A massa seca de raiz de milho é influenciada pela aplicação de pós de rochas. 
As maiores massas secas da parte área de milho são obtidas com uso de pós de rochas de Lourde (R1) e de Cristalândia (R2).

Considerando a escassez de informações científicas sobre os efeitos dos pós de rocha quanto a disponibilidade de nutrientes e seu efeito nas culturas, este trabalho contribui para o aumento da literatura científica atual.

No entanto, sugere-se pesquisas futuras com a aplicação de pós de rochas em sistemas de cultivo a longo prazo e no campo para valida-se o uso de materiais alternativos como fonte de nutrientes, evitando a dependência de fertilizantes químicos na agricultura.

\section{Agradecimentos}

Agradecemos o apoio financeiro da Coordenação de Aperfeiçoamento Pessoal de Nível Superior - Brasil (CAPES) a nome dos Professores Visitantes JGA y AMZ.

\section{Referências}

Aguilera, JG, Zuffo, AM, Ratke, RF, Trento, ACS, Lima, RE, Gris, GA, Morais, KAD, Silva, JX \& Martins, WC. (2020). Influencia de dosis de polvo de basalto sobre cultivares de soya. Revista Research, Society and Development, 9(1), e51973974.

Bhering, LL. (2017). Rbio: A Tool For Biometric And Statistical Analysis Using The R Platform. Crop Breeding and Applied Biotechnology, 17(2), 187-190.

Bhering, L. L. (2017). Rbio: A tool for biometric and statistical analysis using the R platform Rbio: A tool for biometric and statistical analysis using the R platform SOFTWARE/DEVICE RELEASE. Crop Breeding and Applied Biotechnology - Crop Breeding and Applied Biotechnology, 17(17), 187-190.

Brasil. Ministério da Agricultura Pecuária e Abastecimento. (2017). Manual de métodos analíticos oficiais para fertilizantes e corretivos. Brasília: MAPA.

Camargo, C. K., De Resende, J. T. V., Camargo, L. K. P., Figueiredo, A. S. T., \& Zanin, D. S. 
(2012). Produtividade do morangueiro em função da adubaçã o orgânica e com pó de basalto no plantio. Semina:Ciencias Agrarias, 33(SUPPL.1), 2985-2994.

Daryanto, S., Wang, L., \& Jacinthe, P.-A. (2015). Global Synthesis of Drought Effects on Food Legume Production. PloS One, 10(6), e0127401.

Dreyer, I., Gomez-Porras, J. L., \& Riedelsberger, J. (2017). The potassium battery: a mobile energy source for transport processes in plant vascular tissues. In New Phytologist 216(4), 1049-1053.

Fuentes, L. F. G., de Souza, L. C. F., Serra, A. P., Rech, J., \& Vitorino, A. C. T. (2018). Corn agronomic traits and recovery of nitrogen from fertilizer during crop season and off-season. Pesquisa Agropecuaria Brasileira, 53(10), 1158-1166.

Gonzalez Aguilera, J., Mario Zuffo, A., Felippe Ratke, R., Silva Trento, A. C., Lima, R. E., Alves Gris, G., Morais, K. A. D. de, Silva, J. X. da, \& Martins, W. C. (2020). Influencia de dosis de polvo de basalto sobre cultivares de soya. Research, Society and Development, 9(7), 51973974.

Hoagland, D. R., \& Arnon, D. I. (1950). The water culture method for growing plants without soils. Berkeley: California Agricultural Experimental Station.

Jing, J., Christensen, J. T., Sørensen, P., Christensen, B. T., \& Rubæk, G. H. (2019). Long-term effects of animal manure and mineral fertilizers on phosphorus availability and silage maize growth. Soil Use and Management, 35(2), 323-333. doi: 10.1111/sum.12477

Klein, C., \& Dutrow, B. (2012). Manual de ciência dos minerais. Porto Alegre: Bookman.

Li, C., Li, C., Zhang, H., Liao, H., \& Wang, X. (2017). The purple acid phosphatase GmPAP21 enhances internal phosphorus utilization and possibly plays a role in symbiosis with rhizobia in soybean. Physiologia Plantarum, 159(2), 215-227. 
Lu, L., Qiu, W., Gao, W., Tyerman, S. D., Shou, H., \& Wang, C. (2016). OsPAP10c, a novel secreted acid phosphatase in rice, plays an important role in the utilization of external organic phosphorus. Plant Cell and Environment, 39(10), 2247-2259.

Manning, D. A. C., \& Theodoro, S. H. (2018). Enabling food security through use of local rocks and minerals. Extractive Industries and Society.

Menegale, M. L. de C., Castro, G. S. A., \& Mancuso, M. A. C. (2015). Silício: interação com o sistema solo-planta. Journal of Agronomic Sciences, 4(especial), 435-454.

Melo, M. P. De, Caiam, R., Lima, P., Araújo, G., Freitas, D., \& Oliveira, S. (2018). Fontes e doses de fósforo na produção de Panicum maximum cv. Massai Sources and doses of phosphorus in the production of Panicum maximum cv. Massai. Tecnologia \& Ciência Agropecuária, 12(2), 25-35.

Pereira, A., Shitsuka, D., Parreira, F., \& Shitsuka, R. (2018). Metodologia da pesquisa científica. Santa Maria: UAB/NTE/UFSM.

Pragana, R. B., Ribeiro, M. R., Nóbrega, J. C. A., Filho, M. R. R., \& da Costa, J. A. (2012). Qualidade física de Latossolos Amarelos sob plantio direto na região do Cerrado Piauiens. Revista Brasileira de Ciencia Do Solo, 36(5), 1591-1600.

Quaggio, J. . (2000). A acidez e calagem em solos tropicais. Campinas: Instituto Agronômico de Campinas.

Ramirez-Cabral, N. Y. Z., Kumar, L., \& Shabani, F. (2017). Global alterations in areas of suitability for maize production from climate change and using a mechanistic species distribution model (CLIMEX). Scientific Reports, 7(1), 1-13.

Ramos, C. G., Mello, A. G. De, \& Kautzmann, R. M. (2014). Environmental Nanotechnology, Monitoring \& Management A preliminary study of acid volcanic rocks for stonemeal application. Environmental Nanotechnology, Monitoring \& Management, 1(2), 3035 . 
Ratke, R. F., Pereira, H. S., Santos_Junior, J. D. G. dos, Barbosa, J. M., \& Lopes, L. O. (2018). Different limestone particle sizes for soil acidity correction, $\mathrm{Ca}$ and $\mathrm{Mg}$ supply and corn yield. Comunicata Scientiae, 9(2), 175-184.

Ribeiro, S., \& Ranulfo, A. (2010). Rochas silicáticas portadoras de potássio como fontes do nutriente para as plantas solo. Revista Brasileira de Ciência Do Solo, 34(1), 891-897.

Santos, D. R. dos, Gatiboni, L. C., \& Kaminski, J. (2008). Fatores que afetam a disponibilidade do fósforo e o manejo da adubação fosfatada em solos sob sistema plantio direto. Ciência Rural, 38(2), 576-586.

Schaller, J., Faucherre, S., Joss, H., Obst, M., Goeckede, M., Planer-Friedrich, B., Peiffer, S., Gilfedder, B., \& Elberling, B. (2019). Silicon increases the phosphorus availability of Arctic soils. Scientific Reports, 9(1), 1-11.

Silva, F. M. da, Alves, L. S., Botelho Filho, F. B., \& Silva, I. S. (2017). Liquidez dos contratos futuros de milho negociados na BM\&FBOVESPA. Revista de Administração $e$ Negócios Da Amazônia, 9(1), 26.

Silva, F. S. (2011). Manual de análises de solos, plantas e fertilizantes. (2 $2^{\mathrm{a}}$. Brasília: Embrapa.

Srivastava, A. K., Mboh, C. M., Gaiser, T., Kuhn, A., Ermias, E., \& Ewert, F. (2019). Effect of mineral fertilizer on rain water and radiation use efficiencies for maize yield and stover biomass productivity in Ethiopia. Agricultural Systems, 168, 88-100.

Straaten, P. V. A. N. (2006). Farming with rocks and minerals : challenges and opportunities. $78,731-747$.

Swaney, D. P., \& Howarth, R. W. (2019). Phosphorus use efficiency and crop production: Patterns of regional variation in the United States, 1987-2012. Science of the Total Environment, 685, 174-188. 
Teixeira, P. C., Donagemma, G. K., Fontana, A., \& Teixeira, W. G. (2017). Manual de métodos de análises de solos $\left(3^{\mathrm{a}}\right)$. Brasília: Embrapa.

Viadé, A., Fernández-marcos, M. L., Hernández-nistal, J., \& Alvarez, E. (2011). Effect of particle size of limestone on $\mathrm{Ca}, \mathrm{Mg}$ and $\mathrm{K}$ contents in soil and in sward plants. Scientia Agricola, 68(2), 200-208.

Wang, B., Liu, H., Wang, X. H., Li, J. M., Ma, Y. B., Ma, A. X. W., \& Ma, X. W. (2015). Soil phosphorus accumulation model for an arid area of north-western China with 3-year rotation of wheat, maize and cotton. Journal of Agricultural Science, 153(7), 1247-1256.

Wang, Y., \& Wu, W. H. (2017). Regulation of potassium transport and signaling in plants. In Current Opinion in Plant Biology, 39(1), pp. 123-128.

West, P. C., Gerber, J. S., Engstrom, P. M., Mueller, N. D., Brauman, K. A., Carlson, K. M., Cassidy, E. S., Johnston, M., MacDonald, G. K., Ray, D. K., \& Siebert, S. (2014). Leverage points for improving global food security and the environment. Science, 345(6194), 325-328.

\section{Porcentagem de contribuição de cada autor no manuscrito}

Rafael Felippe Ratke - 30\%,

Thatiane Gomes Andrade - 10\%,

Sara Gomes da Rocha-10\%,

Alan de Sousa $-8 \%$

Paulo Vinicius da Silva Dai - 8\%,

Edson Cavalcante da Silva Filho - 8\%

Luiz Carlos Bertolino-8\%

Alan Mario Zuffo - 8\%

Augusto Matias de Oliveira - 5\%

Jorge González Aguilera - 5\% 